v. Meyer: Untersuchung d. aus einigen Saarkohlen etc. 389 zu sein, wenn ich dieses zu ungefähr 2 p.C. von der der Nitrirung unterworfenen Benzoësäure annehme. Es ist dieses allerdings nur eine sehr geringe Ausbeute. Bedenkt man aber, dass auch die Methode ihrer Darstellung aus nitrirter Zimmtsäure verhältnissmässig nur sehr wenig ergiebig zu sein scheint, und dass dieselbe ausserdem jedenfalls viel mehr Zeit in Anspruch nimmt, so möchte es, ganz abgesehen von dem hohen Preise der Zimmtsäure, vorläufig doch unentschieden bleiben, welches Verfahren der Gewinnung dieser Säure den Vorzug verdient.

\title{
Untersuchung der aus einigen Saarkohlen stammenden Gase;
}

ron

\section{Dr. E. จ. Moyer.}

Die mit den Steinkohlen des Saarbeckens in nächstem Zusammenhang stehenden gasförmigen Producte, welche theils als Schlagwetter das Leben des Bergmanns gefabrden, theils noch eingeschlossen in den Kohlen, gefahrlos bleiben, sind bisher kaum einem genaueren Studium unterworfen wurden. G. Bisch of's Analysen ${ }^{1}$ ) von zwei Grubengzsen, welche den Saarkohlen entströmten, müssen bei Vergleich der jetzigen analytischen Hülfsmittel mit denen, welche ihm zu Gebot standen (im Jahre 1840), unbedingt Zweifel erregen; besonders auffallend ist der von ihn beobachtete Gehalt an Aethylen.

Von späteren Untersuchungen natürlicher Grubengass aus dern Saarbezirk habe ich nur zwei Analysen, eine von

1) Edinburgh Now Phil. Journ. 29, 309 and 80, 127. 
Bunsen ${ }^{1}$ ), die andere von Keller ${ }^{2}$ ) auftinden können, welche die Abwesenheit des schweren Kohlenwasserstoffs constatirten.

Um zur Lösang dieser immerhin noch schwebenden Frage womöglich beizutragen, besuchte ich im Juli d. d. die Gruben Gerhard bei Louisenthal und „Kronprinz Friedrich Wilhelm" bei Griesborn, beide in der Nähe von Saarbrücken, welche seit einiger Zeit vorzugsweise mit schlagenden Wettern behaftet waren.

Ich gedenke an dieser Stelle des regen Interesses, mit welchem mir und meinem Unternehmen Herr Oberbergrath Achenbach entgegenkam, und der Liebenswürdigkeit, mit welcher derselbe und die Herren Bergdirectoren mich bei meinem Vorhaben unterstützt haben, mit aufrichtigster Dankbarkeit.

In dem alten Gerhardstollen hatte G. Bischof ein Gas, welches als sog. Bläser aus einer Spalte des Schieferthons austrat, zu seiner Untersuchung aufgefangen. Da zur Zeit meines Besuchs kein Bläser in diesem Bezirk vorhanden war, sammelte ich an einer Stelle, welche vorzugsweise mit Schlagwettern behaftet war, mehrere Proben in Flaschen, welche vor der Einfahrt mit heissem ausgekochtem Wasser gefüllt waren; dieselben wurden vorläufig gut verkorkt und an gefahrloser Stelle versiegelt.

In der Grube Kronprinz Friedrich Wilhelm fand ich in einem Querschlage nach dem Wahlschieder Flötze (in der 5. Tiefbausohle, etwa 133 Meter unter der Tagesoberfläche) einen aus dem sehr dichten Schieferthon im Hangenden mit grosser Gewalt austretenden Bläser vor, welchen ich durch Röhren behufs Aufsammelns strömen liess. Dieselben wurden vorläufig gut geschlossen, un so bald als möglich zugeschmolzen zu werden. Leider verunglückte die erste durch Aufblasen in Folge zu starken Drucks; in der Besorgniss, auch die übrigen einzu-

2) Mitgetheilt Pogg. Ann. 83, S. 252 bei Gelegenheit seiner abhandlung über die Processe der valk. Gesteinsbildungen Islands.

3) Jahresber. 7, 891. 
bussen, begnügte ich mich mit einem Verschluss durch geschmolzenes Wachs, welcher freilich sich nicht als völlig genügend erweisen sollte.

Um ausser diesen an Ort und Stelle gewonnenen Fdnkten auch die in den Koblen dieser beiden Gruben enthaltenen Gase untersuchen zu können, wandte ich mich an die Directoren dieser Werke, die Herren Freund and Maass, welche mit der dankenswerthesten Bereitwilligkeit mir eine Anzahl Proben ans den verschiedenen Flötzen sandten. Auf meine Bitte versahen mich die Herren mit genauen Angaben über Lagerungsverhältnisse, Tiefe etc. dieser Kohlen.

Da mir nicht möglich war, alle Sorten gleichzeitig in Arbeit zu nehmen, mussten die meisten kürzer oder länger liegen (an einem kühlen, trocknen Ort). Ioh werde jedesmal eine ungefähre Angake über die Zeit, welche von der Förderung bis zur Verarbeitung der Kohlen verstrich, beifügen.

Um die Gase aus den Kohlen zu treiben und aufzufangen, bediente ich mich der in diesem Journal [2] 5, 146 beschriebenen Methode. Trügt man Sorge, dass die Gase stets heisses, ansgekochtes Wasser durchstreichen, so kann man auf einigermassen zuverlüssige Kohlensäurebestimmungen rechnen. So dürften die Fehler, welche durch das als Sperrflüssigkeit benutzte Wasser verursacht werden könnten, auf ein geringes Maass beschränkt sein.

\section{Kohlen aus der Grube Kronprinz Friedrich} Wilhelm bei Griesborn.

\section{A. Wahlschieder Flötz.}

Za dem Bereich dieser Grube gehört das Wahlschieder Flötz; da mit diesem der vorhin erwähnte Bläser in unzweifelhafter Beziehung stand, so möge das, was ich über dessen Beschaffenheit feststellen konnte, vorangeschickt werden.

Meine Befürehtung, dass wegen mangelhaften Verschlusses der Röhren atmosphärische Luft eingedrungen 
392 v. Meyer: Untersuchng der aus einigen

sei, wurde bestätigt. Dennoch theile ich hier zwei Analysen des Gases mit, weil noch genug brennbare Bestandtheile vorhanden waren, um einen Rückschluss auf die Beschaffenheit des ursprünglichen Gases machen zu können.

Analyse (1):

\begin{tabular}{l|c|c|c|c|c}
\hline & $\begin{array}{c}\text { Beob. } \\
\text { Vol. }\end{array}$ & Temp. & Barom. & $\begin{array}{c}\text { Queaks.- } \\
\text { Walle üb. d. }\end{array}$ & $\begin{array}{c}\text { Corrig. } \\
\text { Vol }\end{array}$ \\
\hline Angew. Vol. (feucht) & 141,5 & 13,0 & 748,9 & 24,2 & 96,37 \\
N.Abs.d.CO (trocken) & 140,3 & 13,1 & 745,1 & 25,4 & 96,35 \\
N.Abs. von O (trocken) & 121,3 & 13,4 & 743,2 & 44,1 & 80,84
\end{tabular}

\begin{tabular}{l|c|c|c|c|c} 
Angew. Vol. (fourht) & 95,2 & 14,1 & 746,4 & 450,5 & 24,89 \\
Nach Zul. v. O (feucht) & 165,3 & 14,2 & 745,0 & $\mathbf{3 9 1 , 1}$ & 53,73 \\
N. Verpuffung (feucht) & 132,2 & 13,9 & 744,9 & 424,3 & 38,85 \\
N. Abs.der CO (trook.) & 112,75 & 13,2 & 742,1 & 443,6 & 32,10 \\
N.Zul. von H (trocken) & 205,2 & 13,9 & 740,6 & $\mathbf{3 5 1 , 3}$ & $\mathbf{7 6 , 0 2}$ \\
N. Verpuffung (feucht) & 121,4 & 14,0 & 740,4 & 435,1 & 33,88
\end{tabular}

b.

\begin{tabular}{|c|c|c|c|c|c|}
\hline Angew. Vol. (feucht) & 117,2 & 14,7 & 754,9 & 439,0 & 33,74 \\
\hline Nach Zul. v. O (feuoht) & 215,4 & 14,8 & 755,2 & 340,0 & 81,06 \\
\hline N. Verpuffung (foucht) & 180,2 & 14,8 & 755,4 & 375,4 & 62,82 \\
\hline N. $\mathrm{Abs}$ der $\mathrm{CO}_{2}$ (trock) & 156,85 & 13,3 & 754,1 & 398,7 & $\mathbf{5 3 , 1 8}$ \\
\hline N.Zul. von H (trocken) & 390,5 & 13,5 & 752,8 & 163,3 & 219,36 \\
\hline N. Verpuffung (feucht) & 292,3 & 13,5 & 750,5 & 262,9 & 182,61 \\
\hline
\end{tabular}

Die erhaltenen Zahlen sprechen mit genügender Sicherheit dafüir, dass der brennbare Bestandtheil Grubengas ohne Beimengung anderer Kohlenwasserstoffe ist. Nach den beiden Analysen ist die procentische Zusammensetzung:

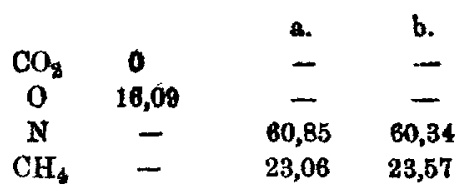

Bringt man die 16,09 r.C. Sauerstoff in atmosphärischer Laft entsprechende Stickstoffmenge 60,67 p.C. in Abrechnung, bleibt reines Grubengas abrig. Deraus 
darf jedoch keinenfalls der Schluss gezogen werden, der Bläser habe keine anderen Bestandtheile enthalten. ${ }^{1}$ )

In unmittelbarer Nähe von der Stelle, an welcher der Bläser austrst, wurden an einer niedrigen Arbeitsstrecke Kohlen frisch gebrochen. (Zwei Hauer hatten neben einander Platz.) Um zu erfahren, ob die Luft in dieser Aushöhlung wesentlich verändert sei, oder ob die trefflichen Ventilationsvorrichtungen der Grube an dieser ungünstig gelegenen Stelle vollständig wirksam seien, sammelte ich kurz nach einender ${ }^{2}$ ) zwei Proben in Arzeneiflaschen, welche zuror mit ausgekochtem Wasser gefüllt waren. Dieselben warden mit Kork versehlossen und so. bald als möglich versiegelt.

Analyse der zuerst gesammelten Luft:

\begin{tabular}{|c|c|c|c|c|c|}
\hline (2) & $\begin{array}{l}\text { Beob. } \\
\text { Vol. }\end{array}$ & Temp. & Barom. & $\begin{array}{l}\text { Quecks.: } \\
\text { Säule üb. d. } \\
\text { Wanne. }\end{array}$ & $\begin{array}{l}\text { Corrig. } \\
\text { Vol. }\end{array}$ \\
\hline Angew. Vol. (feucht) & 166,0 & 12,6 & 747,1 & 32,6 & 111,70 \\
\hline N. Abs. der $\mathrm{CO}_{2}$ (trock.) & 162,2 & 13,3 & 748,0 & 35,0 & 110,30 \\
\hline Angew. Vol. (feucht) & 90,95 & 13,1 & 744,9 & 419,9 & 27,23 \\
\hline $\begin{array}{c}\text { Nach Verpuffung mit } \\
\text { Knallgas (feucht) }\end{array}$ & 87,8 & 13,2 & 744,2 & 424,0 & 25,87 \\
\hline N.Abs. der $\mathrm{CO}_{2}$ (trock.) & 88,45 & 13,1 & 744,0 & 427,4 & 25,21 \\
\hline N. Zul. van H (trocken) & 115,05 & 13,1 & 746,9 & 394,4 & 38,70 \\
\hline N. Verpuffung (feucht) & 92,85 & 13,6 & 746,2 & 417,1 & 28,08 \\
\hline
\end{tabular}

Aus diesen Zahlen berechnet sich, wenn man dio Annahme den erhaltenen Werthen gemäss macht, die geringe Menge verbrannten Gases sei Grubengas, folgende Zusammensetzung:

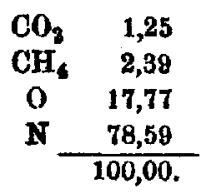

1) Durch Diffusion der Luft kann in manchen Fällen der Sanerstoffgehalt darselben vergrössort werden, wie z. B. Graham in auffallendster Weise beim Durchgang von Luft durch Kautschnk beobach. tot hat. varlassen.

7) Die beiden Arbeiter hatten einige Zeit rorhor diesen Platz 
394 v. Meyer: Untersuchung der aus einigen

Dass die bei der Berechnung gemachte Voraussetzung richtig ist, geht aus der folgenden Analyse hervor, welche mit der später gesammelten Luft ausgefiihrt wurde; der Sauerstoff derselben wurde durch Absorption und auf eudiometrischem Wege bestimmt.

\begin{tabular}{|c|c|c|c|c|c|}
\hline (3) & $\begin{array}{l}\text { Beob. } \\
\text { Vol. }\end{array}$ & Temp. & Barom. & \begin{tabular}{|c|} 
Quecks.- \\
Säule üb. d. \\
Wanne.
\end{tabular} & $\begin{array}{l}\text { Corrig. } \\
\text { Vol. }\end{array}$ \\
\hline
\end{tabular}

\begin{tabular}{l|r|r|r|r|r} 
Angow. Vol. (teucht) & 214,3 & 10,5 & 734,9 & 11,9 & 147,24 \\
N.Abs. der $\mathrm{CO}_{2}$ (trock.) & 205,0 & 9,6 & 735,1 & 21,0 & 141,42 \\
\hline
\end{tabular}

Absorption mit pyrogallnssanrem Kali.

\begin{tabular}{l|l|l|l|l|l} 
Angew. Vol. (trocken) & 117,4 & 9,4 & 747,0 & 40,2 & 80,22 \\
N. $\Delta$ bs. des $O$ (trock.) & 100,1 & 8,4 & 753,8 & 58,2 & 67,55 \\
\hline \multicolumn{1}{|c|}{$\beta$. }
\end{tabular}

Eudiometrische Bestimmnng.

\begin{tabular}{c|l|l|l|l|l} 
Angew. Vol. (feucht) & 154,85 & 10,3 & 735,4 & $\mathbf{3 6 2 , 4}$ & $\mathbf{5 4 , 2 6}$ \\
$\begin{array}{c}\text { Nach Verpuffung mit } \\
\text { Kuallgas (feucht) }\end{array}$ & 148,8 & 10,5 & $\mathbf{7 3 5 , 5}$ & $\mathbf{3 6 8 , 3}$ & $\mathbf{5 1 , 2 6}$ \\
N.Abs.der CO (trook.) $_{\text {) }}$ & 144,85 & 10,0 & $\mathbf{7 2 9 , 2}$ & $\mathbf{3 7 2 , 1}$ & 49,90 \\
N.Zul.von H (trocken) & 184,85 & 10,5 & $\mathbf{7 3 1 , 0}$ & $\mathbf{3 3 1 , 9}$ & $\mathbf{7 1 , 0 4}$ \\
Nach Verpuffg.(feucht) & 156,2 & 10,5 & 731,3 & $\mathbf{3 6 0 , 8}$ & $\mathbf{5 4 , 3 0}$
\end{tabular}

b. (2. Portion.)

\begin{tabular}{l|c|c|c|c||c} 
Angew. Vol. (feucht) & 136,75 & 8,4 & 747,1 & 19,6 & 95,15 \\
N.Abs. derCO (trock.) & 130,35 & 8,3 & 749,8 & 26,1 & $\mathbf{9 1 , 5 5}$
\end{tabular}

Die Zusammensetzung dieser Luft ist daher folgende:

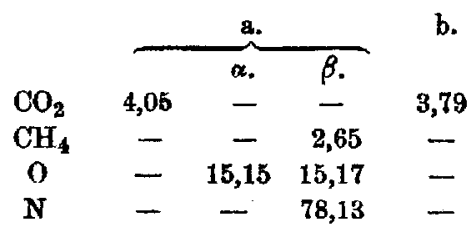

Die gewonnenen Zahlen bedürfen kaum eines Commentars; sie dürften beweisen, dass an ungüinstig gelegenen Punkten in Kohlenbergwerken, auch wenn in den Haupt- 
strecken derselben der trefflichste Wetterzug herrscht, die Luft stagnirt und durch Anwesenheit von Menschen, Brennen der Grubenlichter und sicherlich auch durch den Einfluss der Kohle auf den Sauerstoff auffallend schnell verändert, $d . h$. verschlechtert wird. Volle Beweiskraft haben natiurlich diese Zahlen nicht, weil sie noch vereinzelt dastehen. Eine grüdliche chemische Untersuchung der Grubenluft, bei welcher die technischen Rücksichten selbstverständlich nicht vernachlüssigt werden dürfen, wäre sehr wiinschenswerth im Interesse des gesammten Betriebs und der Gesundheit des Arbeitspersonals.

Die höchst verdienstvolie Abhandlung des Bergraths Pfühler „Wetterführung auf der Königl. Steinkohlengrube Sulzbach-Altenwald bei Saarbrücken" (veröffentlicht in der Zeitschrift für das Berg-, Hütten" und Salinenwesen) stellt die Schwierigkeiten in das klarste Licht, mit welchen die Ventilation ausgedehnter Grubengebäude verknüpft ist, und lässt keinen Zweifel darüber, dass man gerade in den Gruben des Saardistricts Alles aufbietet, um die gesundheitlich nothwendige Luftcirculation zu ermöglichen. Es wäre von hohem Interesse gewesen, wenn gleichzeitig mit den zahlreichen, von Herrn Pfähler mitgetheiluen Versuchen, welche die Geschwindigkeit des Wetterstroms und die davon abhängigen Luftquantitäten feststellen sollten, Analysen der unter wechselnden Bedingungen und an verschiedenen Punkten aufgesammelten Grubenluft ausgeführt worden wären. Man hätte sich gewiss ein vollständigeres Bild von der Wirksamkeit der Ventilationsvorrichtungen in jener Grube machen können. $\mathrm{Ob}$ noch besondere lokale Ventilatoren in Thätigkcit treten. müssen und können, um ungünstig gelegene Strecken mit frischer Luft zu versorgen, ist dann Sache der Bergtechnik.

Wie ich höre, werden regelmässige Untersuchungen, bei welchen auch der Kohlensäuregehalt der Grubenluft Berücksichtigung findet, schon seit einiger /eit in der bayerischen Grube Bexbach angestellt, welche auch zum Saardistrikt gehört. Soviel mir bekannt, ist bisher niemals 
ein so hoher Kohlensäure- und so geringer Sauerstoffgehalt, wie Analyse (3) answeist, beobaohtet worden.

Vergleicht man die Analysen (2) und (3) in Bezug auf jene beiden Bestandtheile, so erkennt man, dass die Summe derselben nahezu die gleiche ist; man erhält nach (2) 19,02, wach (3) 19,08 (wenn man 3,92 $\mathrm{CO}_{2}$ im Mittel annimmat). Da nun in atmosphärischer Luft dieser Procentzahl (19,05 O) nur 71,85 p.C. Stickstoff entsprechen, aber 78,59 , resp. 78,13 p.C. gefunden wurden, so wird dieser Sauerstoffmangel dureh die bekannte Eigenschaft der Steinkohlen, Sauerstoff zu absorbiren, crklärt werden mïssen.

Aus dem Wahlschieder Flötze waren mir zwei Kohlenproben gesandt worden, welche ich sofort nach ihrer Ankunft (2 Wochen nachdem sie gebrochen waren) untersuchte. Die erste war einer dem Bläser benachbarten Stelle entnommen und trug folgende Bezeichnung:

Aus der Grundstrecke der 5. Tiefbausohle des Ostfeldes, 120 Meter tief.

Kohle regelmässig geschichtet, von verwittertem Aussehen, reich an Schwefelkies. Gasentwickelung mässig.

Analyse des Gases:

\begin{tabular}{|c|c|c|c|c|c|}
\hline (4) & $\begin{array}{l}\text { Beob. } \\
\text { Vol. }\end{array}$ & Temp. & Barom. & \begin{tabular}{|} 
Quecks. \\
Säule üb. d. \\
Wanne.
\end{tabular} & $\begin{array}{c}\text { Corrig. } \\
\text { Vol. }\end{array}$ \\
\hline Angew. Vol. (feucht) & 187,4 & 14,4 & 744,1 & 28,4 & 131,92 \\
\hline N.Abs.der $\mathrm{CO}_{2}$ (trock.) & 180,3 & 14,4 & 750,1 & 45,6 & 120,66 \\
\hline Naeh Abs. d. O (trock.) & 180,4 & 15,5 & 750,4 & 45,5 & 120,34 \\
\hline Angew. Vol. (feucht) & 82,75 & 15,6 & 747,9 & 434,2 & 23,52 \\
\hline N. Zul. v. Luft (feucht) & 308,9 & 15,6 & 747,8 & 207,7 & 153,97 \\
\hline N. Zul. von $\theta$ (feucht) & 374,85 & 16,0 & 746,9 & 141,3 & 209,67 \\
\hline N. Verpuffung (feucht) & 333,6 & 15,4 & 746,2 & 182,7 & 173,85 \\
\hline N.Abs.der $\mathrm{CO}_{2}$ (trock.) & 307,1 & 15,1 & 748,2 & 208,8 & 155,52 \\
\hline N. Zul. von $\mathbf{H}$ (trocken) & 500,55 & 15,4 & 743,7 & 16,6 & 338,38 \\
\hline N. Verpuffung (teuoht) & 364,85 & 15,2 & 744,0 & 151,8 & 200,18 \\
\hline
\end{tabular}

Die exhaltenen Zahlen sprechen dafiir, dass neben Grubengas eine kleine Menge eines Kohlenwasserstoflis der 
Reihe $\mathrm{Cn}_{2 n+2}$ vorhanden ist; nimmt man $\mathrm{C}_{2} \mathrm{H}_{6}$ an, so hat das Gas folgende Zusammensetzung:

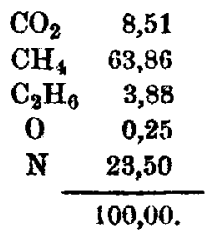

\begin{tabular}{|c|c|c|}
\hline & $\begin{array}{c}\text { Berechnete Werthe } \\
\qquad 17,46\end{array}$ & $\begin{array}{r}\text { Gefundene V } \\
17,46\end{array}$ \\
\hline & $1,0 \mathrm{C}_{2} \mathrm{H}_{6}+16,46 \mathrm{CH}_{4}$ & \\
\hline $\mathrm{CO}_{2}$ & 18,46 & 18,33 \\
\hline Contral & ition $\quad 35,42$ & 35,82 \\
\hline Verora & uchter 036,42 & 36,89 \\
\hline
\end{tabular}

Etwa 8 Wochen später wurde dieselbe Kohle in Arbeit genommen; ausser einer Analyse des Gases wurde auch die Menge desselben bestimmt. $100 \mathrm{Grm}$. lieferten $42 \mathrm{Cc}$.

Die Analyse ergab folgende Zahlen:

\begin{tabular}{l|c|c|c|c|c}
\hline \multicolumn{1}{c|}{ (5) } & $\begin{array}{c}\text { Beob. } \\
\text { Vol. }\end{array}$ & Temp. & Barom. & $\begin{array}{r}\text { Quecks.- } \\
\text { Sinle üb.d. } \\
\text { Wanne. }\end{array}$ & $\begin{array}{c}\text { Corrig. } \\
\text { Val. }\end{array}$ \\
\hline Angew. Vol. (feucht) & 111,4 & $\mathbf{9 , 3}$ & $\mathbf{7 4 4 , 8}$ & $\mathbf{4 1 , 4}$ & $\mathbf{7 4 , 8 4}$ \\
N.Abs. der CO (trock.) & $\mathbf{7 6 , 2}$ & $\mathbf{8 , 5}$ & $\mathbf{7 4 8 , 6}$ & $\mathbf{7 6 , 8}$ & $\mathbf{4 9 , 6 5}$ \\
N. Abs. d. O (trocken) & $\mathbf{7 5 , 4}$ & $\mathbf{8 , 5}$ & $\mathbf{7 4 8 , 8}$ & $\mathbf{7 8 , 2}$ & $\mathbf{4 9 , 0 4}$ \\
Angew. Vol. (feucht) & 101,35 & $\mathbf{9 , 4}$ & $\mathbf{7 4 9 , 1}$ & $\mathbf{4 5 7 , 0}$ & $\mathbf{2 7 , 7 6}$ \\
N. Zul. v. Luft (feucht) & $\mathbf{2 5 3 , 5}$ & 9,6 & $\mathbf{7 4 9 , 1}$ & $\mathbf{3 0 4 , 1}$ & $\mathbf{1 0 6 , 8 0}$ \\
NachZul.von O (feucht) & $\mathbf{2 9 7 , 7}$ & $\mathbf{9 , 6}$ & $\mathbf{7 4 9 , 0}$ & $\mathbf{2 5 9 , 7}$ & $\mathbf{1 3 8 , 1 6}$ \\
N. Verpuffung (feucht) & $\mathbf{2 6 2 , 8}$ & $\mathbf{9 , 6}$ & $\mathbf{7 4 9 , 0}$ & $\mathbf{2 9 4 , 9}$ & $\mathbf{1 1 3 , 0 3}$ \\
N.Abs. d. CO (trocken) & $\mathbf{2 3 8 , 3}$ & $\mathbf{8 , 8}$ & $\mathbf{7 4 7 , 5}$ & $\mathbf{3 1 9 , 4}$ & $\mathbf{9 8 , 8 3}$ \\
N.Zul. von H (trocken) & 337,55 & $\mathbf{9 , 3}$ & $\mathbf{7 4 7 , 5}$ & $\mathbf{2 2 1 , 2}$ & $\mathbf{1 7 1 , 8 0}$ \\
N. Verpuffung (feacht) & $\mathbf{2 5 8 , 6 5}$ & $\mathbf{9 , 5}$ & $\mathbf{7 4 7 , 2}$ & $\mathbf{3 0 0 , 1}$ & $\mathbf{1 0 9 , 5 6}$
\end{tabular}

Das Gas enthält in 100 Theilen:

$$
\begin{array}{cr}
\mathrm{CO}_{3} & 83,66 \\
\mathrm{CH}_{4} & 24,08 \\
\mathrm{C}_{2} \mathrm{H}_{0} & 4,72 \\
\mathrm{O} & 0,81 \\
\mathrm{~N} & 36,73 \\
\hline & 100,00
\end{array}
$$




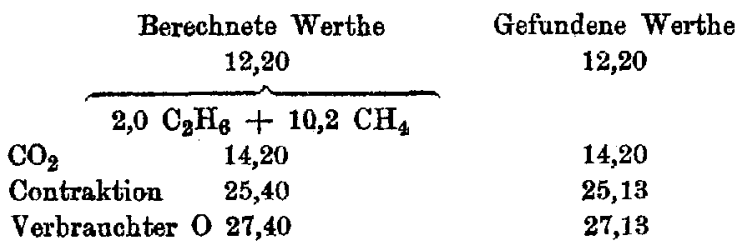

Die Resultate der Analysen (4) und (5) haben insoweit etwas Ueberraschendes, als das Gas einen wenn auch geringen Gehalt an einem höheren Kohlenwasserstoff aufweist, während der Bläser nach der Analyse (1) nur Grubengas enthielt.

Ich glaube die Erklärung dieser Erscheinung in der Leichtigkeit, mit welcher das Grubengas von der Kohle abgegeben wird, zu erblicken, während Aethylwasserstott in gewisser Weise fester gebunden ist. Ich erinnere in dieser Beziehung an meine mit einigen Zwickaner Kohlen gemachten Versuche ${ }^{1}$, welche die eben besprochene Verschiedenheit beider Gase illustriren.

Vergleicht man die Analyse (4) und (5) mit einander, so zeigt sich, dass der Gehalt an $\mathrm{C}_{2} \mathrm{H}_{6}$ relativ zugenommen hat, während eine bedeutende Abnahme des $\mathrm{CH}_{4}$ erfolgt ist. Nach (4) enthalten 100 Theile der Kohlenwasserstoffe

$$
\begin{array}{r}
5,7 \mathrm{C}_{2} \mathrm{H}_{6} \text { und } 94,3 \mathrm{CH}_{4} \text {, nach (5) } \\
16,4 \Rightarrow, \quad, \quad 83,6 \text {, }
\end{array}
$$

Mit der Verminderung der Kohlenwasserstoffe in (5) steht die Zunahme der $\mathrm{CO}_{2}$ in engem Zusammenhange, eine Beobaohtung, welche ich schon oft bei meinen früheren Untersuchungen gemacht habe.

Wir sind in gewisser Weise berechtigt, aus der Grösse und Schnellinkeit dieser Zunahme auf die grössere oder geringere Verwitterungsfähigkeit der Kohlen zu schliessen.

Die zweite demselben Flötze entnommene Kohle war auf der Grundstrecke der 7. Tiefbausohle des neuen West feldes (152 Meter tief) gebrochen. Ihr Aussehen war im

1) Nachtrag zo den Versuchen mit Zwickauer Kohlen. Dies. Journ. [2], 5, 421. 
Allgemeinen dem der anderen Kohle ähnlich; nur war sie weniger hart, und arm an Sehwefellies. Sie wurde gleichzeitig mit jener untersucht.

Analyse des Gases:

\begin{tabular}{|c|c|c|c|c|c|}
\hline (6) & $\begin{array}{c}\text { Beob. } \\
\text { Vol. } \\
\end{array}$ & Temp. & Barom. & \begin{tabular}{|c|} 
Quecks.- \\
Saule iüb. d. \\
Wanne.
\end{tabular} & $\begin{array}{c}\text { Corrig. } \\
\text { Vol. }\end{array}$ \\
\hline Angew. Vol. (feucht) & 185,1 & 14,7 & 743,7 & 37,9 & 121,81 \\
\hline N.Abs. der $\mathrm{CO}_{2}$ (trock.) & 173,0 & 13,9 & 754,0 & 50,0 & 115,90 \\
\hline Nach Abs.d.O (trocken) & 172,7 & 13,4 & 753,3 & 48,9 & 115,96 \\
\hline Angow. Vol (feucht) & 89,8 & 14,0 & 754,0 & 469,1 & 21,37 \\
\hline N. Tul. v. Luft (feucht) & 266,5 & 14,2 & 754,0 & 291,2 & 114,18 \\
\hline Nach Zul. v. O (feucht) & 308,85 & 14,2 & 754,0 & 248,6 & 144,83 \\
\hline N. Verpuffung (feucht) & 262,8 & 14,3 & 753,9 & 295,0 & 111,58 \\
\hline N.Abs.der $\mathrm{CO}_{2}$ (trock.) & 233,2 & 14,8 & 755,9 & 325,4 & 95,23 \\
\hline N.Zul. von $H$ (trocken) & 306,6 & 15,2 & 756,5 & 252,9 & 146,27 \\
\hline N. Verpuffung (feucht) & $\mathbf{2 3 8 , 8}$ & 15,2 & 757,3 & 320,9 & 95,80 \\
\hline
\end{tabular}

Die erhaltenen Zahlen zeigen, dass in diesem Gase als brennbarer Bestandtheil nur Grubengas vorhanden war. In 100 Theilen sind enthalten:

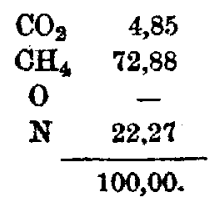

Was die Lagerungsverhältnisse des Wahlschieder Flötzes anbetrifft, so besitze ich darüber folgende Angaben: Im Hangenden und Liegenden ist dasselbe ron festem geschlossenem Schiefer umgeben; es hat einen regelmässigen Verlauf, ist also nicht durch Sprünge verworfen. Die untersuchten Kohlen stammen von neu gebauten Strecken.

\section{B. Schwalbacher Flötz.}

Aus derselben Grube standen mir noch vier Kohlenproben, welche verschiedenen Abtheilungen, resp. Arbeitspunkten des Schwalbacher Flötzes entnommen waren, 
400 v. Meyer: Untersuchung der aus einigen

zu Gebot. Die dasselbe begleitenden Gesteine sind wesentlich die nämlichen, wie bei dem Wahlschieder Flötze; auch sein Verlauf ist ein ungestörter. Die Proben I und III sind Abbaufeldern, welche schon seit einigen Jahrev nusund vorgerichtet sind, II und IV neuen Strecken entnommen.

1. Aus den Abbauarbeiten über der 5. Tiefbausohle, westl. Wetterstrecke 6, Ostfeld. (Aus einer Tiefe von 116 Met.)

Die Kohle war deutlich gesehichtet, nicht sehr hart und verhältnissmässig arm an Schwefelkies. (Zeit der Verarbeitung: 3 Wochen nach der Förderung.)

Bei der Analyse wurden folgende Zahlen erhalten:

\begin{tabular}{c|c|c|c|c|c}
\hline \hline$(7)$ & $\begin{array}{c}\text { Beol. } \\
\text { Vol. }\end{array}$ & Temp. & Barom. & $\begin{array}{c}\text { Quecks. } \\
\text { Saule uib. d. } \\
\text { Wanne. }\end{array}$ & $\begin{array}{c}\text { Corrig. } \\
\text { Vol. }\end{array}$ \\
\hline \hline Angew. Vol. (feucht) & 165,7 & 14,8 & 756,4 & 22,7 & 113,36 \\
N.Abs.der CO (trock.) & 162,5 & 13,2 & 762,2 & 64,3 & 108,18
\end{tabular}

Absorption mit pyrogallussanrem Kali.

\begin{tabular}{l|r|r|r|r|r} 
Angew. Vol. (trocken) & 100,5 & 14,5 & 761,7 & 72,7 & 65,76 \\
Nach Abs. (trocken) & 109,4 & 11,5 & 741,6 & 118,1 & 65,46
\end{tabular}

\begin{tabular}{l|r|r|r|r|r} 
Angew. Yol. (feucht) & $\mathbf{8 6 , 6 5}$ & 11,2 & $\mathbf{7 4 3 , 6}$ & $\mathbf{4 3 2 , 1}$ & 25,10 \\
N. Zul. v. Luft (feucht) & 337,55 & 11,2 & $\mathbf{7 4 4 , 2}$ & 180,2 & $\mathbf{1 7 9 , 6 7}$ \\
Nach Zul. v. O (feucht) & $\mathbf{3 7 3 , 8}$ & $\mathbf{1 1 , 4}$ & $\mathbf{7 4 4 , 4}$ & $\mathbf{1 4 4 , 1}$ & $\mathbf{2 1 1 , 7 8}$ \\
N. Verpuffung (feucht) & $\mathbf{3 3 9 , 1 5}$ & $\mathbf{1 1 , 6}$ & $\mathbf{7 4 4 , 2}$ & $\mathbf{1 7 9 , 4}$ & $\mathbf{1 8 0 , 4 4}$ \\
N.Abs.der CO (trock.) & 317,65 & $\mathbf{9 , 6}$ & $\mathbf{7 3 4 , 2}$ & $\mathbf{2 0 0 , 9}$ & $\mathbf{1 6 3 , 6 5}$ \\
Nach Zul.v.H (trocken) & 414,2 & 8,8 & $\mathbf{7 4 9 , 3}$ & 104,9 & $\mathbf{2 5 8 , 5 8}$ \\
Nach Varpuffg. (feucht) & 315,45 & $\mathbf{9 , 6}$ & $\mathbf{7 5 0 , 7}$ & $\mathbf{2 0 3 , 5}$ & $\mathbf{1 6 4 , 0 4}$
\end{tabular}

Die gewonnenen analytischen Daten sprechen für folgende procentische Zusammensetzung des Gases:

\begin{tabular}{cr}
$\mathrm{CO}_{2}$ & 4,57 \\
$\mathrm{CH}_{4}$ & 51,84 \\
$\mathrm{C}_{2} \mathrm{H}_{6}$ & 5,92 \\
$\mathrm{O}$ & 0,26 \\
$\mathrm{~N}$ & 37,41 \\
\hline & 100,00
\end{tabular}




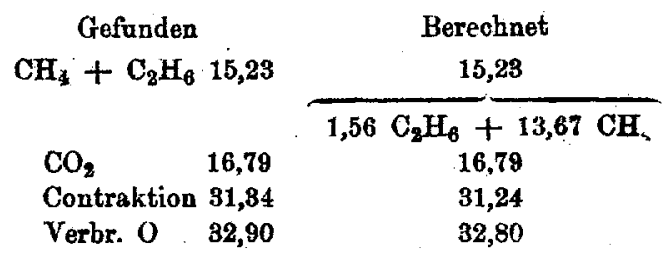

5-6 Wochen, nachdem dies Gas aufgesammelt war, wurde ein anderes Stück derselben Kohle auf die eingeschlossenen Gase geprüft. $100 \mathrm{Grm}$. lieferten $32 \mathrm{Cc}$. Gas.

Da in die eudiometrische Bestimmung sich ein Fehler eingeschlichen haben muss, theile ich nur die absorptiometrische ausfübrlich mit, welche dadurch interessant ist, dass sie eine ausserordentliche Kohlensäurezunahme constatirt.

\begin{tabular}{c|r|r|r|r|r}
\hline (8) & $\begin{array}{c}\text { Beob. } \\
\text { Vol. }\end{array}$ & Temp. & Barom. & $\begin{array}{c}\text { Quecks.- } \\
\text { Saule üb.d. } \\
\text { Wanne. }\end{array}$ & $\begin{array}{c}\text { Corr. } \\
\text { Vol. }\end{array}$ \\
\hline \hline Angew. Vol. (feucht) & 149,6 & 10,5 & 747,2 & 79,2 & 94,86 \\
N.Abs.d.CO (trocken) & 81,8 & 9,8 & 748,9 & 147,5 & 47,50 \\
NachAbs.d.O(trocken) & 80,8 & $-9,5$ & 739,6 & 148,2 & 46,18
\end{tabular}

Nach der allerdings unzuverlässigen endiometrisehen Analyse wurden 15,04 p.C. Kohlenwasserstoffe gefunden. Wäre dieser Gehalt der richtige, so hätte das Gas folgende Zusammensetzung:

$$
\begin{array}{cr}
\mathrm{CO}_{2} & -49,95 \\
\left(\mathrm{C}_{8} \mathrm{H}_{\mathrm{a}}+\mathrm{CH}_{4}\right) & -(15,04) \\
\mathrm{O} & -1,39 \\
(\mathrm{~N}) & -(33,62) \\
\hline & 100,00
\end{array}
$$

Der Zuwachs an Kohlensäure in (8) ist bej Vergleich mit (7) so bedeutend, dass man auf eine höchst lebhafte Oxydation im Innern der Kohlen schliessen muss. Leider habe ich versäumt, den Gasgehalt der frischeren Kohle zu bestimmen; ich zweifle nicht, dass derselbe erheblich grösser -gewesen ist, als die Gasmenge der zuletzt untersuchten Probe.

Journ. f. prakt. Chemle [2] Bd. 6. 
II. Aus der Grundstrecke der 7. Tiefbausohle bei Wetterstrecke 6, Ostfeld (156 Met. Tiefe).

Die Kohle, bröcklich, von verwittertem Aussehn, mit Schwefelkies reichlich durchsetzt, wurde 3 bis 4 Wochen, uachdem sie gebrochen war, untersucht; 100 Grm. ergaben 19 Cc. Gas.

Analyse des Gases:

\begin{tabular}{|c|c|c|c|c|c|}
\hline$(9)$ & $\begin{array}{l}\text { Beob. } \\
\text { Vol. }\end{array}$ & Temp. & Barom. & $\begin{array}{l}\text { Quecks. } \\
\text { Sämle üb. d. } \\
\text { Wanne. }\end{array}$ & $\begin{array}{l}\text { Corrig. } \\
\text { Vol. }\end{array}$ \\
\hline Ange & 185,7 & 9,6 & 734,8 & 10,0 & 93,83 \\
\hline N.Abs.d. $\mathrm{CO}_{2}$ (trocken) & 82,2 & 8,7 & 748,3 & 32,2 & 57,13 \\
\hline N. Abs. d. 0 (trocken) & 86,7 & 9,3 & 746,6 & 78,2 & 56,04 \\
\hline
\end{tabular}

a.

\begin{tabular}{l|r|r|r|r|r} 
Angew. Vol. (feucht) & 99,05 & $\mathbf{8 , 6}$ & $\mathbf{7 5 4 , 6}$ & $\mathbf{4 5 5 , 8}$ & $\mathbf{2 7 , 9 4}$ \\
Nach Zul. v. O (feucht) & $\mathbf{1 8 8 , 6}$ & $\mathbf{8 , 7}$ & $\mathbf{7 5 4 , 6}$ & $\mathbf{3 6 5 , 1}$ & $\mathbf{6 9 , 6 6}$ \\
N. Verpuffung (foucht) & 130,2 & $\mathbf{9 , 0}$ & $\mathbf{7 5 4 , 4}$ & 425,1 & 40,43 \\
N. Abs. der COg (trock.) & 80,4 & $\mathbf{8 , 5}$ & $\mathbf{7 5 0 , 7}$ & $\mathbf{4 7 5 , 9}$ & $\mathbf{2 1 , 4 3}$ \\
N. Zul. v. H. (trocken) & 209,7 & $\mathbf{7 , 9}$ & $\mathbf{7 4 8 , 0}$ & $\mathbf{3 4 5 , 5}$ & $\mathbf{8 2 , 0 3}$ \\
N. Verpuffung (feucht) & 178,1 & $\mathbf{8 , 6}$ & $\mathbf{7 4 6 , 5}$ & $\mathbf{3 7 6 , 8}$ & $\mathbf{6 2 , 4 0}$
\end{tabular}

\section{Absorption mit Schwefelsänre.}

\begin{tabular}{l|l|l|l|l|l} 
Angew. Vol. (trocken) & 41,35 & 8,2 & 745,8 & 111,4 & 25,47 \\
Nach Abs. (trocken) & 41,45 & 8,3 & 743,5 & 111,3 & 25,43
\end{tabular}

b.

\begin{tabular}{l|r|r|r|r|r} 
Angew. Vol. (feucht) & $\mathbf{8 1 , 3}$ & 8,9 & $\mathbf{7 4 4 , 5}$ & $\mathbf{4 7 4 , 4}$ & $\mathbf{2 0 , 6 0}$ \\
N. Zul. v. Luft (foucht) & 207,5 & 9,1 & 744,7 & $\mathbf{3 4 7 , 0}$ & $\mathbf{7 8 , 1 3}$ \\
N. Zul. von O (feucht) & $\mathbf{2 4 6 , 9}$ & $\mathbf{9 , 1}$ & $\mathbf{7 4 5 , 0}$ & $\mathbf{3 0 7 , 7}$ & $\mathbf{1 0 2 , 4 3}$ \\
N. Verputfung (feucht) & $\mathbf{2 1 2 , 4}$ & $\mathbf{9 , 4}$ & $\mathbf{7 4 5 , 1}$ & $\mathbf{3 4 2 , 2}$ & 80,92 \\
N. A bs. der CO (trock.) & 184,1 & 10,3 & $\mathbf{7 4 9 , 9}$ & $\mathbf{3 7 0 , 5}$ & $\mathbf{6 7 , 3 1}$ \\
N. Zul. von $\mathrm{H}$ (trocken) & $\mathbf{2 4 5 , 3}$ & $\mathbf{1 1 , 1}$ & $\mathbf{7 5 1 , 1}$ & 308,5 & $\mathbf{1 0 4 , 3 3}$ \\
NachVerpuffg. (fencht) & 196,3 & 11,1 & $\mathbf{7 5 1 , 2}$ & 357,5 & $\mathbf{7 2 , 4 2}$
\end{tabular}

Die eben mitgetheilten Analysen beweisen, dass kein durch rauchende Schwefelsäure absorbirbarer Bestandtheil in dem Gase enthalten war. Denn abgesehen davon, dass keine Absorption wahrzunehmen war, stimmen die vor und 
nach der Behandlung mit Schwefelsäure gemachten eudiometrisehen Bestimmungen a. und b. untereinander überein. Nach a. wurden gefunden 28,38 p.C., nach b. 27,85 p.C. Kohlenwasserstoffe, im Mittel 28,12 p.C. Die erhaltenen analytischen Daten führen zu der folgenden (mittleren) procentischen Zusammensetzung:

\begin{tabular}{|c|c|}
\hline $\mathrm{CO}_{2}$ & 38,05 \\
\hline $\mathrm{CH}_{4}$ & 15,06 \\
\hline $\mathrm{C}_{2} \mathrm{H}_{6}$ & 13,06 \\
\hline 0 & 1,16 \\
\hline $\mathrm{N}$ & 32,67 \\
\hline $\mathrm{C}_{n} \mathrm{H}_{2 n}$ & - \\
\hline
\end{tabular}

\begin{tabular}{|c|c|c|c|c|}
\hline & \multicolumn{2}{|c|}{ 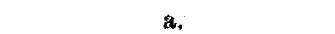 } & \multicolumn{2}{|c|}{ b. } \\
\hline & Berechnet & Gefunden & Berechnet & Gefunden \\
\hline $\mathrm{CH}_{4}+\mathrm{C}_{2} \mathrm{H}_{6}$ & 12,92 & 13,05 & 9,54 & 9,44 \\
\hline Gebildete $\mathrm{CO}_{3}$ & 18,92 & 19,00 & 13,97 & 13,61 \\
\hline Contraktion & 28,84 & 29,23 & 21,29 & 21,51 \\
\hline Verbr. 0 & 34,84 & 35,18 & 25,72 & 25,68 \\
\hline
\end{tabular}

III. Aus den Abbauarbeiten über der 5. Tiefbausoble des newen Westfeldes (Tiefe: 110 Meter).

Die Kohle war von ziemlich harter, regelmässig geschichteter Struktur; 100 Grm. derselben enthielten $25 \mathrm{Cc}$. Gas; (sie hatte etwa 5 Wochen gelegen, ehe sie in Arbeit genommen wurde.)

Von dem Gas wurde eine eudiometrische Bestimmung vor Absorption mit Schwefelsäure a. und eine nach derselben $b$. ausgeführt.

\begin{tabular}{c|c|c|c|c|c}
\hline (10) & $\begin{array}{c}\text { Beob. } \\
\text { Vol. }\end{array}$ & Temp. & Barom. & $\begin{array}{c}\text { Quecks. } \\
\text { Wale üb.d. } \\
\text { Wanne. }\end{array}$ & $\begin{array}{c}\text { Corrig. } \\
\text { Vol. }\end{array}$ \\
\hline Angew. Vol. (feucht) & 129,9 & 12,4 & 746,9 & 41,9 & 86,27 \\
N. Abs. d. CO (trock.) & $95,3$. & 12,8 & 745,3 & $\mathbf{7 7 , 2}$ & $\mathbf{6 0 , 8 2}$ \\
N. Abs. d. O. (trocken) & 91,5 & 13,0 & $\mathbf{7 4 6 , 9}$ & $\mathbf{8 1 , 1}$ & $\mathbf{5 8 , 1 5}$
\end{tabular}




\begin{tabular}{|c|c|c|c|c|}
\hline $\begin{array}{l}\text { Beob. } \\
\text { Vol. }\end{array}$ & & Barom. & $\begin{array}{l}\text { Quecks.- } \\
\text { Säule üb.d. } \\
\text { Wanne. }\end{array}$ & $\begin{array}{l}\text { Coirig. } \\
\text { Vol. }\end{array}$ \\
\hline
\end{tabular}

a.

\begin{tabular}{l|c|c|c|r|r} 
Angew. Voi. (feucht) & 72,3 & 12,8 & 741,0 & 436,7 & 20,26 \\
N. Zul. von O (feucht) & 130,55 & 11,8 & 738,8 & 377,9 & 43,88 \\
N. Zul. v. Luft (feucht) & 246,75 & 11,9 & 738,5 & 261,3 & 110,38 \\
N. Verpuffung (feucht) & 225,55 & 11,8 & 736,7 & 282,6 & 95,96 \\
N. Abs. d. CO (trock.) & 204,0 & 11,0 & 745,0 & 308,8 & 86,52 \\
Nash Zul.v. H. (trock.) & 301,4 & 10,0 & 748,9 & 206,8 & 157,62 \\
N. Verpuffung (feucht) & 222,75 & 10,4 & 747,3 & 286,5 & 96,86
\end{tabular}

Absorption mit Schwefelsänre.

\begin{tabular}{l|r|r|r|r|r} 
Augew. Vol. (trocken) & 55,2 & 10,1 & $\mathbf{7 3 5 , 5}$ & $\mathbf{1 1 3 , 5}$ & $\mathbf{3 3 , 1 0}$ \\
Nach Abs. (trocken) & $\mathbf{5 5 , 5}$ & $\mathbf{9 , 8}$ & $\mathbf{7 3 0 , 0}$ & $\mathbf{1 1 3 , 2}$ & $\mathbf{3 3 , 0 5}$
\end{tabular}

b.

\begin{tabular}{l|r|r|r|r|r} 
Angew. Vol. (feucht) & 106,3 & 10,5 & 731,0 & 449,0 & 27,89 \\
N. Zul. v. Iuft (feucht) & 223,7 & 10,5 & $\mathbf{7 3 1 , 3}$ & $\mathbf{3 3 0 , 9}$ & $\mathbf{8 4 , 2 1}$ \\
N. Zul. v. O (feucht) & 244,2 & 9,9 & $\mathbf{7 4 6 , 6}$ & $\mathbf{3 1 0 , 1}$ & $\mathbf{1 0 0 , 7 2}$ \\
N. Verpuffung (feucht) & 211,4 & 10,0 & $\mathbf{7 4 6 , 7}$ & $\mathbf{3 4 3 , 0}$ & $\mathbf{8 0 , 4 5}$ \\
N. Abs. d. CO (trook.) & 182,8 & 8,6 & 753,7 & 372,6 & 67,54 \\
N. Zul.vou H (trocken) & $\mathbf{2 3 2 , 8}$ & 8,8 & $\mathbf{7 5 3 , 8}$ & 321,9 & $\mathbf{9 7 , 4 1}$ \\
N. Yerpuffung (feucht) & $\mathbf{2 1 7 , 3}$ & $\mathbf{9 , 0}$ & $\mathbf{7 5 3 , 5}$ & $\mathbf{3 3 7 , 3}$ & $\mathbf{8 5 , 7 5}$
\end{tabular}

Auch diese Analysen (10) führen zu dem Resultat, welches (9) ergeben hat. Das Gas ist frei von einem Kohlenwasserstoff der Reihe $\mathrm{C}_{\mathfrak{n}} \mathrm{H}_{2 n}$, wie aus der Uebereinstimmung von a. und $b$. hervorgeht.

Procentische Zusammensetzung des Gases:

$$
\begin{array}{cccc}
\mathrm{CO}_{2} & 29,50 & - & \text { b. } \\
\mathrm{C}_{2} \mathrm{H}_{6}+\mathrm{CH}_{4} & - & 21,92 & - \\
\mathrm{O} & 3,09 & - & - \\
\mathrm{N} & - & 45,49 & 46,15
\end{array}
$$

Legen wir die Analyse a. zu Grunde, nach welcher die gefundenen 21,92 p.C. sich auflösen in $9,48 \mathrm{C}_{2} \mathrm{H}_{6}$ und $12,44 \mathrm{CH}_{4}$ und berechnen $b$. nach $a$, so erhalten wir allseitig gut stimmende Werthe, wie folgende Zusammenstellung lehrt: 


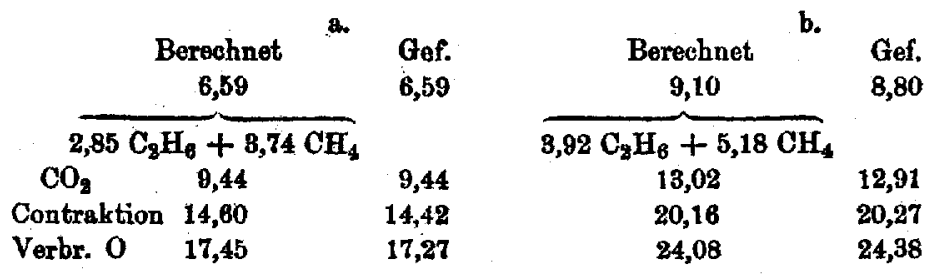

IV. Aus der (im Abteufen begriffenen) Wetterstrecke Nr. I unterhalb der 7. Tiefbausohle des neuen Westfeldes (Tiefe: 148 Meter.

Verarbeitung der Kohle: etwa 4 Wochen nach ihrer Förderung. Dieselbe, unregelmässig geschichtet, zeigt auf ihren Bruchflächen zahlreiche Verwitterungsprodukte (von Schwefelkies); 100 Grm. lieferten 22,5 Cc. Gas.

Analyse des Gases:

\begin{tabular}{|c|c|c|c|c|c|}
\hline (11) & $\begin{array}{l}\text { Beob. } \\
\text { Vol. }\end{array}$ & Temp. & Barom. & $\begin{array}{l}\text { Quecks.- } \\
\text { Säule üb. d. } \\
\text { Wanne. }\end{array}$ & $\begin{array}{c}\text { Corrig. } \\
\text { Yol. }\end{array}$ \\
\hline Angew. Vol. (feucht) & 147,1 & 12,5 & 742,1 & 15,7 & $100,6 \mathrm{~B}$ \\
\hline N. $\mathrm{Abs}, \mathrm{d}, \mathrm{CO}_{8}$ (trock) & 186,1 & 11,5 & 741,6 & 36,4 & 85,34 \\
\hline N. Abs, d. O (trocken) & 125,4 & 11,7 & 741,9 & 37,2 & 84,75 \\
\hline Angew. Vol (feucht) & 112,15 & 11,2 & 743,6 & 441,0 & 31,54 \\
\hline N.Zul. v. Luft (feucht) & 359,6 & 11,2 & 744,2 & 191,6 & 187,47 \\
\hline N. Znl. v. $O$ (fouaht) & 406,7 & 11,4 & 744,4 & 144,2 & 230,38 \\
\hline N. Yerpuffung (fouoht) & 364,4 & 11,6 & 744,2 & 186,4 & 191,42 \\
\hline N. $\triangle b_{3}$ d. $\mathrm{CO}_{8}$ (trook) & 338,65 & 9,6 & 734,2 & 212,3 & 170,69 \\
\hline
\end{tabular}

Obwohl die Sauerstoffbestimmung verunglückte und somit die Analyse unvollständig blieb, glaube ich doch den in diesem Gase enthaltienen brennbaren Bestandtheil als Grubengas ansprechen zu dürfen, da der erhaltenen Contraktion 38,96 - 19,48 $\mathrm{CO}_{2}$ entsprechen sollen, während 19,73 gefunden wurden. Demnach enthält das Gas in 100 Theilen:

\begin{tabular}{cr}
$\mathrm{CO}_{3}$ & $\mathbf{1 5 , 2 2}$ \\
$\mathrm{CH}_{3}$ & $\mathbf{5 2 , 1 2}$ \\
$\mathrm{O}$ & $\mathbf{0 , 5 6}$ \\
$\mathbb{N}$ & 32,10 \\
\hline & $100,00$.
\end{tabular}

Hiermit wurden die Versuche mit Kohlen aus der Grube Kronprin z abgeschlossen. 
406 v. Meyer: Untersuchung der aus einigen

II. Kohlen der Grube Geislautern.

Die mir von Herrn Dir. Maas s freundlichst gemachten Mittheilungen über die Flötze Nr. 4, 5 und 6 diescr Grube, welchen die Proben I, II und III entnommen waren, lasse ich hier folgen:

Die Flotze sind vielfach verworfen und in der Lagerung yostört, sio sind auf beiden Seiten von Schieferthon begleitet. Die Proben I und III stammen von neuen Strecken.

I. Flötz Nr. 4, aus der Grundstrecke der 2. Tiefbausohle zwischen den Sprüngen Kastor und Pollux (aus einer Tiefe von 88 Meter).

Die Kohle, von sehr verwittertem Ausseben, aber deutlicher Schichtung, wurde etwa 6 Wochen, nachdem sie gebrochen war, verarbeitet. $100 \mathrm{Grm}$. gaben 26,0 Cc. Gas.

Analyse des Gases:

\begin{tabular}{|c|c|c|c|c|c|}
\hline$(12)$ & $\begin{array}{l}\text { Beob. } \\
\text { Vol. }\end{array}$ & Temp. & Barom. & $\begin{array}{c}\text { Quecks.- } \\
\text { Säule üb. d. } \\
\text { Wanne. }\end{array}$ & $\begin{array}{l}\text { Corrig. } \\
\text { Vol. }\end{array}$ \\
\hline A ngew. Vol. (feucht) & 142,4 & 11,4 & 735,8 & 7,2 & 98,23 \\
\hline N. Abs. d. $\mathrm{CO}_{2}$ (trock.) & 117,3 & 10,4 & 746,9 & 48,4 & 79,38 \\
\hline N. Abs. d. O (trocken) & 114,4 & 9,8 & 746,8 & 35,8 & 78,43 \\
\hline Angew. Vol. (feucht) & 94,6 & 10,0 & 739,8 & 458,8 & 24,77 \\
\hline N. Zul. v. Luft (feucht) & 305,7 & 10,1 & 739,0 & 246,3 & 142,58 \\
\hline N. Zul. จ. $O$ (feucht) & 350,5 & 10,2 & 738,1 & 201,5 & 178,16 \\
\hline N. Verpuffung (feucht) & 318,7 & 10,2 & 737,2 & 233,1 & 152,02 \\
\hline N. Abs. d. $\mathrm{CO}_{2}$ (trock.) & 296,1 & 10,3 & 741,4 & 256,2 & 138,45 \\
\hline N. Zul. v. H (trocken) & 439,25 & 10,5 & 735,0 & 112,5 & 263,31 \\
\hline N. Vorpuffung (foucht) & 334,7 & 10,7 & 735,0 & 217,1 & 163,68 \\
\hline
\end{tabular}

Die erhaltenen Daten stimmen für folgende procentische Zusammensetzung:

\begin{tabular}{cr}
$\mathrm{CO}_{2}$ & 19,19 \\
$\mathrm{CH}_{4}$ & 37,94 \\
$\mathrm{C}_{2} \mathrm{H}_{6}$ & $\mathbf{3 , 2 2}$ \\
$\mathrm{O}$ & 0,97 \\
$\mathrm{~N}$ & $\mathbf{3 8 , 6 8}$ \\
\hline & 100,00
\end{tabular}




\begin{tabular}{lcc}
\multicolumn{1}{c}{$\begin{array}{c}\text { Berechnet } \\
12,77\end{array}$} & $\begin{array}{c}\text { Gefunden } \\
12,67\end{array}$ \\
$\mathrm{CO}_{2} \mathrm{C}_{2} \mathrm{H}_{6}+11,77 \mathrm{CH}_{4}$ & \\
Conbraktion & 26,77 & 13,57 \\
Verbr. $\mathrm{O}$ & 27,04 & 26,14 \\
& & 27,04
\end{tabular}

II. Flötz Nr. 5, aus den Abbauarbeiten über der Theilungsstrecke Nr. 1 der 2. Tiefbausohle südlich des Kastorsprunges (Tiefe: 40 Met.)

Unregelmässig geschichtete, schwefelkiesreiche Kohle (beinahe Russkohle). 100 Grm. lieferten 21,2 Ce. Gas (Zeit der Verarbeitung: 8 Wochen etwa nach Förderung).

Analyse des Gases:

\begin{tabular}{|c|c|c|c|c|c|}
\hline (13) & $\begin{array}{l}\text { Beob. } \\
\text { Vol. }\end{array}$ & Temp. & Barom. & $\begin{array}{l}\text { Quechs.- } \\
\text { Suiule üb. d. } \\
\text { Wanne. }\end{array}$ & $\begin{array}{c}\text { Corrig. } \\
\text { Vol. }\end{array}$ \\
\hline Augrew. Vol. (foncht) & 134,4 & 8,5 & 745,1 & 31,0 & 92,00 \\
\hline N. $\mathrm{Abs}$, d. $\mathrm{CO}_{2}$ (trocks) & 90,4 & 8,3 & 749,8 & 75,2 & 59,19 \\
\hline N. Abs. d. O (trocken) & 87,4 & 8,4 & 745,1 & 78,1 & 56,56 \\
\hline Angew. Vol. (fencht) & 78,75 & 9,3 & 745,0 & 438,0 & 22,72 \\
\hline N. Zual. v. Luft (feucht) & 189,75 & 9,5 & 745,0 & 326,1 & 75,19 \\
\hline N. Zul. v, O (feucht) & 217,25 & 9,6 & 744,8 & 298,7 & 91,76 \\
\hline N. Verpuffung (foucht) & 200,65 & 9,7 & 74,4 & 315,1 & 81,44 \\
\hline N. Abs, d. $\mathrm{CO}_{2}$ (trock.) & 185,75 & 8,9 & 748,6 & $3 \$ 0,1$ & 75,28 \\
\hline N. Zul. v. $\mathrm{H}$ (trocken) & 288,75 & 9,0 & 748,2 & 226,8 & 145,75 \\
\hline N. Verpuffung (foucht) & 225,3 & 9,0 & 748,1 & 290,0 & 98,07 \\
\hline
\end{tabular}

Absorption mit ranch. Schwefelsänre. .

\begin{tabular}{l|l|l|l|l|l} 
Angew. Vol. (trocken) & 43,5 & $\mathbf{7 , 9}$ & $\mathbf{7 4 8 , 0}$ & 110,0 & $\mathbf{2 6 , 9 8}$ \\
Nach Abs. (trocken) & 43,1 & $\mathbf{6 , 4}$ & $\mathbf{7 5 1 , 1}$ & 110,4 & $\mathbf{2 7 , 0 1}$
\end{tabular}

Nach dieser Analyse enthielt das Gas keinen durch Schwefelsäure absorbirbaren Bestandtheil, wohl aber neben Grubengas Acthylwasserstoff. 
408 จ. Meyer: Untersuchung der aus einigen

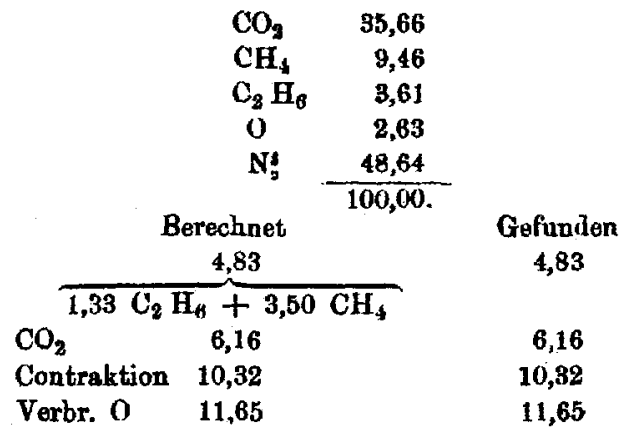

III. Flötz Nr. 6, aus der Grundstrecke der 2. Tiefbausohle, zwischen den Sprüngen Kastor und Pollux (Tiefe: 96 Meter).

Die deutlich geschichtete, nicht sehr harte Kohle wurde etwa 6 Wochen nach ihrer Förderung untersucht; $100 \mathrm{Grm}$. lieferten $53 \mathrm{Cc}$. Gas.

Analyse desselben:

\begin{tabular}{|c|c|c|c|c|c|}
\hline (14) & $\begin{array}{l}\text { Beob. } \\
\text { Vol. }\end{array}$ & Temp. & Barom. & $\begin{array}{l}\text { Quecka.: } \\
\text { Säule üb.d. } \\
\text { Wanne. }\end{array}$ & $\begin{array}{l}\text { Corrig. } \\
\text { Vol. }\end{array}$ \\
\hline Angew. Vol. (feucht) & 127.2 & 13,4 & 748,2 & 92,4 & 78,12 \\
\hline N. Abs. d. $\mathrm{CO}_{2}$ (trock.) & 96,9 & 12,8 & $\mathbf{7 4 5 , 3}$ & 121,8 & 57,71 \\
\hline N. $\mathbf{A b s} . \mathrm{d} . \mathrm{O}$ (trooken) & 96,7 & 13,2 & 746,5 & 181,1 & 56,77 \\
\hline Angew. Vol. (fenobt). & 123,0 & 12,8 & 741,0 & 488,6 & 34,24 \\
\hline N. Zul. จ. O (feucht) & 204,4 & 11,8 & 738,8 & $\mathbf{3 5 7 , 0}$ & T2,70 \\
\hline N. Zul. v. Luft (feacht) & 319,0 & $11, \theta$ & 738,6 & 242,1 & 148,56 \\
\hline N. Verpuffung (feucht) & 244,5 & 11,8 & 786,7 & 316,9 & 95,98 \\
\hline N. $\mathrm{Abs}$, d. $\mathrm{CO}_{2}$ (trook.) & 192,5 & 11,0 & 745,0 & 368,0 & 68,76 \\
\hline
\end{tabular}

Aus diesen Daten wird folgende procentische Zusammensetzung berechnet:

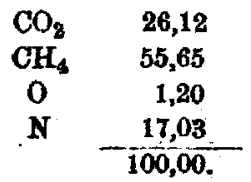

III. Kohlen der Grube Gerhard bei Lonisenthal.

Aus dieser Grube standen mir drei Proben zur Verfügung, welche den Flötzen Anna (I), Sophie (II) und 
Max (III) des Albertschachtes entnommen waren. Die äussere Beschaffenheit dieser Kohlen wair wesentlich dieselbe. Alle zeigten kaum eine Spur von Schichtung, waren sehr hart und von glänzendem, muschligem Bruch. Sämmtliche Proben wurden etwa 4 bis 5 Wochen, nachdem sie gebrochen waren, in Arbeit genommen.

Zur Charakteristik der drei Flötze mögen folgende, mir giutigst mitgetheilte Angaben dienen: „Die Flötze I und II haben Schieferthon und Sandstein zum Hangenden und Liegenden; II lagert in einem saigern Abstand von 7 Lachter unter 1 . Das Flötz III liegt 6 Lachter unter II und hat festes Conglomerat zum Hangenden, Schieferthon und Sandstein zum Liegenden. - Sämmtliche Flötze sind stark mit sehlagenden Wettern behaftet und vielfach durch Sprünge verworfen. - Die Flötze I bis III liegen cirea 100 Lachter weiter im Liegenden der Gerhardgrubenflötze, wo s. Z. G. Bi sch of einen Wetterbläser aufsammelte."

In demselben Schachte hatte ich, wie schon in der Einleitung bemerkt, an einer Stelle, welche starke Ansammlungen von Schlagwettern zeigte, diese aufgefangen. Die Analyse derselben, welche entseheiden sollte, ob Grubengas allein oder auch andere Kohlenwasserstoffe vorhanden seien, will ich zuerst mittheilen.

\begin{tabular}{c|c|c|c|c|c}
\hline (15) & $\begin{array}{c}\text { Beob. } \\
\text { Vol. }\end{array}$ & Temp. & Barom. & $\begin{array}{c}\text { Quecks. } \\
\text { Saule üb.d. } \\
\text { Wanne. }\end{array}$ & $\begin{array}{c}\text { Corrig. } \\
\text { Vol. }\end{array}$ \\
\hline Angew. Vol. (foucht) & 107,0 & 11,4 & $\mathbf{7 5 0 , 0}$ & 52,0 & $\mathbf{7 0 , 6 7}$ \\
N. Abs. d. CO, (trook.) & 105,3 & 11,2 & 748,3 & 5,0 & $\mathbf{7 0 , 2 3}$
\end{tabular}

\section{Absorption mit pyrogallussanron Kali.}

\begin{tabular}{l|l|l|l|l|l} 
Angew. Vol. (trocken) & 137,2 & 11,5 & 745,8 & 21,2 & 95,40 \\
Niwoh Abs. (trooken) & 120,4 & 11,8 & 747,1 & 38,4 & 81,96
\end{tabular}

\begin{tabular}{|c|c|c|c|c|c|}
\hline Angew. Vol. (fouoht) & 82,95 & 12,2 & 748,1 & $\mathbf{4 3 5 , 8}$ & 28,96 \\
\hline N. Zul v. O (fenoht) & $180,8 b$ & 12,2 & 748,1 & 387,6 & 43, \\
\hline N. Verpufiung (fouoht) & 100,75 & 12,2 & 748,2 & 418,0 & 30,8 \\
\hline $\mathrm{N}, \mathrm{Abs}, \mathrm{d}, \mathrm{CO}_{8}$ (trook.) & 82,75 & 12,0 & 746,2 & 495,8 & 4,5 \\
\hline N. Zul. $\mathbf{H}$ (trocken) & 199,5 & 12,8 & 746,2 & 878,9 & 9,0 \\
\hline N. Verpuffung (foucht) & 94,25 & 12,4 & 746,4 & 484,5 & 28,0 \\
\hline
\end{tabular}


410 v. Meyer: Untersuchung der aus einigen

Das Gas bestand in 100 Theilen aus:

\begin{tabular}{cr}
$\mathrm{CO}_{2}$ & 0,62 \\
$\mathrm{CH}_{4}$ & 22,52 \\
0 & 14,01 \\
$\mathrm{~N}$ & 62,85 \\
\hline & 100,00
\end{tabular}

Die gewonnenen analytischen Daten lassen keinen Zweifel darüber, dass nur Grubengas ohne Beimengung anderer Kohlenwasserstoffe in dem schlagenden Wetter vorhanden war.

\begin{tabular}{ccc}
\multicolumn{2}{c}{ Gefunden } & Berechnet \\
$\mathrm{CH}_{\mathrm{i}}$ & $\mathbf{6 , 3 2}$ & $\mathbf{6 , 3 2}$ \\
$\mathrm{CO}_{2}$ & $\mathbf{6 , 2 3}$ & $\mathbf{6 , 3 2}$ \\
Contraction & $\mathbf{1 3 , 0 1}$ & $\mathbf{1 2 , 6 4}$ \\
Verbr. 0 & 12,92 & 12,64
\end{tabular}

Was das Verhältniss des Sauerstoffs zu dem Stickstoff in dern Gase anbelangt, so finden wir auch hier, wie in den Analysen (2) und (3) der Grubenluft oinen erheblichen Ueberschuss ron Stickstoff: ein Moment, welcbes bei späteren Untersuchungen von Grubenluft ganz besondere Beachtung verdient. (Gefunden wurden 62,85 p.C. $N$. welchen für atmosphärische Iuft 16,65 p.C. $O$ entsprechen würden, während 14,01 erhalten wurden.)

Was dic Kohlen dieser Flötze betrifft, so erstreckt sich die Aehnlichkeit untereinander nicht nur auf ihre Structur; sondern auch auf die Qualität der eingeschlossenen Gase.

Die untersuchten 3 Proben waren aus einer Tiefe von circa 150 Meter genommen.

I. $100 \mathrm{Grm}$. lieferten $188 \mathrm{Cc}$. Gas.

Analyse des Gases:

\begin{tabular}{c|c|c|c|c|c}
\hline (16) & $\begin{array}{c}\text { Beob.- } \\
\text { Vol. }\end{array}$ & Temp. & Barom. & $\begin{array}{c}\text { Quecks." } \\
\text { Wale üb.d. } \\
\text { Wanne. }\end{array}$ & $\begin{array}{c}\text { Corrig.- } \\
\text { Vol. }\end{array}$ \\
\hline Angew. Vol. (fencht) & 146,8 & 8,2 & 746,5 & 25,7 & 101,57 \\
N.Abs. der CO (trock.) & 146,5 & 8,2 & 739,2 & 25,3 & 101,54 \\
N.Abs. des O (trocken) & 146,6 & 8,3 & 739,1 & 25,3 & 101,58
\end{tabular}


Absorption mit raueh. Sehwefelsäure.

\begin{tabular}{l|l|l|l|l|l|l} 
Angew. Vol. (trocken) & $\mathbf{1 1 4 , 5 5}$ & $\mathbf{8 , 5}$ & $\mathbf{7 3 9 , 0}$ & $\mathbf{6 0 , 8}$ & $\mathbf{7 5 , 3 5}$ \\
Nach Absorpt. (trook.) & $\mathbf{1 1 4 , 1 5}$ & $\mathbf{8 , 8}$ & $\mathbf{7 4 0 , 6}$ & $\mathbf{5 9 , 6}$ & $\mathbf{7 5 , 3 0}$
\end{tabular}

Eudometrisehe Bestimmang.

\begin{tabular}{l|l|l|l} 
Angew. Vol. (feucht) & 69,8 & 8,6 & 743,5
\end{tabular}

\begin{tabular}{l|l|l}
$44,8,2$ & 19,42
\end{tabular}

\begin{tabular}{l|l|l|l|l|l} 
N. Zul. v. Luft (feucht) & 287,45 & 9,1 & 744,7 & 230,1 & 140,76
\end{tabular}

\begin{tabular}{l|l|l|l|l|l} 
Nach Zul. v. $\mathrm{O}$ (feucht) & 327,6 & 9,1 & 745,0 & 190,0 & 173,23
\end{tabular}

\begin{tabular}{l|l|l|l|l|l} 
Nach Verpuffg. (feucht) & $\mathbf{2 8 2 , 9 5}$ & $\mathbf{9 , 4}$ & $\mathbf{7 4 5 , 1}$ & $\mathbf{2 3 4 , 7}$ & $\mathbf{1 3 7 , 2 1}$
\end{tabular}

\begin{tabular}{l|l|l|l|l|l} 
N.Abs.der $\mathrm{CO}_{2}$ (trock.) & 255,1 & 10,3 & 749,9 & 262,3 & 119,86
\end{tabular}

\begin{tabular}{l|l|l|l|l|l} 
NachZul.von H (trock.) & 331,5 & 11,1 & 751,1 & 186,1 & 179,99
\end{tabular}

\begin{tabular}{l|l|l|l|l|l} 
Nach Verpuffg. (feucht) & $\mathbf{2 4 9 , 0}$ & $\mathbf{1 1 , 1}$ & $\mathbf{7 5 1 , 2}$ & $\mathbf{2 8 6 , 5}$ & $\mathbf{1 1 3 , 1 5}$
\end{tabular}

Zusammensetzung des Gases:

\begin{tabular}{|c|c|c|}
\hline $\mathrm{CO}_{2}$ & - & Spur \\
\hline $\mathrm{CH}_{\mathbf{q}}$ & - & 91,76 \\
\hline 0 & - & 0 \\
\hline $\mathbf{N}$ & $\cdots$ & 8,24 \\
\hline $\mathrm{C}_{\mathrm{n}} \mathrm{H}_{\mathrm{gn}}$ & - & 0 \\
\hline
\end{tabular}

II. $100 \mathrm{Grm}$. lieferten $158 \mathrm{Cc}$. Gas.

Die Analyse ergab folgende Zahlen:

\begin{tabular}{|c|c|c|c|c|c|}
\hline (17) & $\begin{array}{l}\text { Beob.- } \\
\text { Vol. }\end{array}$ & Temp. & Barom. & $\begin{array}{c}\text { Quecks.- } \\
\text { Säule üb.d. } \\
\text { Wanne. } \\
\end{array}$ & $\begin{array}{l}\text { Corrig.- } \\
\text { Vol. }\end{array}$ \\
\hline Angew. Vol. (feucht) & 133,4 & 11,6 & 745,9 & 32,0 & 90,05 \\
\hline N.Abs. d. $\mathrm{CO}_{2}$ (trocken) & 130,6 & 11,6 & 747,5 & 41,9 & 88,40 \\
\hline Angew. Vol. (feucht) & 116,35 & 12,0 & 747,9 & 439,2 & 33,25 \\
\hline N. Zul. v. Luft (feucht) & 445,0 & 12,1 & 768,1 & 109,2 & 267,78 \\
\hline Nach Zul. v. O (feucht) & 480,3 & 12,1 & 748,1 & 73,9 & 305,25 \\
\hline NachVerpuffg. (feucht) & 420,85 & 12,1 & 748,2 & 132,9 & 243,73 \\
\hline N.Abs.d. $\mathrm{CO}_{2}$ (trocken) & 385,4 & 11,9 & 746,2 & 167,8 & 213,57 \\
\hline Naoh Zul.v.H.(trocken) & 456,0 & 12,3 & 746,2 & 97,3 & 283,15 \\
\hline NachVerpuffg. (feucht) & 882,0 & 12,4 & 746,4 & 170,9 & 206,46 \\
\hline
\end{tabular}

Das Gas enthielt demnach in 100 Theilen:

$$
\begin{array}{rr}
\mathrm{CO}_{2} & 1,83 \\
\mathrm{CH}_{4} & 90,79 \\
\mathbf{N}^{*} & 7,88 \\
\hline & 100,00
\end{array}
$$


III. Aus $100 \mathrm{Grm}$. wurden $180 \mathrm{Cc}$. Gas erhalten.

Analyse des Grases:

\begin{tabular}{|c|c|c|c|c|c|}
\hline (18) & $\begin{array}{c}\text { Beob.- } \\
\text { Vol. }\end{array}$ & Terap. & Barom. & $\begin{array}{c}\text { Queoks.: } \\
\text { Qüule üb. d. } \\
\text { Wanne. }\end{array}$ & $\begin{array}{c}\text { Corrig. } \\
\text { Vol }\end{array}$ \\
\hline Angew. Vol. (feucht) & 179,7 & 11,1 & 750,9 & 41,8 & 120,76 \\
\hline N.A.bo.d. $\mathrm{CO}_{2}$ (trocken) & 173,0 & 11,0 & 752,1 & 47,0 & 117,26 \\
\hline Angew. Vol (feucht) & 90,4 & 11,5 & 750,9 & 464,4 & 23,98 \\
\hline N. Zul. v. Luft (feucht) & 345,5 & 11,4 & 750,4 & 208,0 & $176,58^{-}$ \\
\hline Nach Zul. v. $O$ (feucht) & 404,25 & 11,4 & 748,3 & 148,5 & 228,88 \\
\hline Nach Verpuffg. (feucht) & 355,6 & 11,7 & 748,1 & 197,3 & 184,86 \\
\hline N.Abs. d. $\mathrm{CO}_{2}$ (trooken) & 326,55 & 11,7 & 745,8 & 227,3 & 162,36 \\
\hline N.Zul. ron FI (trocken) & 448,75 & 11,4 & 747,1 & 103,9 & 277,08 \\
\hline Nach Verpuffg. (feucht) & 323,6 & 11,8 & 747,5 & 230,1 & 157,31 \\
\hline
\end{tabular}

100 Theile des Gases bestanden aus:

$$
\begin{array}{r}
\mathrm{CO}_{2} \\
\mathrm{CH}_{4} \\
\mathbf{N}
\end{array}
$$

Die Gase aus allen 3 Proben besassen einen, wenn auch nicht starken, aber specifisch scharfen Geruch. Durch die Analysen war jedoch keinerlei fremder Bestandtheil aufzufinden. Die Zahlenwerthe stimmen jedesmal für reines Grubengas.

Man wird durch Quantität und Qualität der in diesen Kohlen eingeschlossenen Gase an die von mir nntersuchten Kohlen des Durham-Distrikts ${ }^{2}$ ) erinnert. Wie bei diesen geht die sehr bedeutende Gasmenge dieser Sasrkohlen mit dem reiehen Gehalt an Grubengas Hand in Hand. Besonders beschtenswerth ist, dass dieselben dio Structur, Härte etc. mit jenen englischen Kohlen gemein haben. Ohne Zweifel ist die äussere Beschaffenheit von wesentlichster Bedeutung für die Menge der eingesohlossenen Gase.

Es schien mir von einigem Interesse, das pecifische Gewicht gerade dieser Kohlen aus der Grube Gerhard, und

3) Dies Journ. (2) 5, 410 . 
zwar in frischem und entgastem Zustande, zu bestimmen. Zu diesem Zwecke bediente ich mich eines cylindrischen Gefässes von Messingblech (Inhalt circa $20 \mathrm{Cc}$.), dessen Boden und gut schliessender Deckel aus feinstem Drahtnetz bestanden. Die zu prüfenden Kohlen wurden in kleinen Stücken in diesem Körbchen gewogen (jedesmal etwa $12 \mathrm{Grm}$.$) . Da die Wage mit Genanigkeit Differenzen von$ $0,05 \mathrm{Grm}$. angab, sind die gefundenen spec. Gewichte nur bis zur zweiten Decimale brauchbar. An dem Bügel, mittelst dessen das Gefäss an den Wagebalken aufgehängt warde, war eine Marke angebracht, welche mit dem Niveau des Wassers zusammenfiel, wenn die Wage im Gleichgewicht war. Zunächst wurde das spec. Gewicht der resp. Kohle in Wasser von $20^{\circ}$ bestimmt, nachdem das Körbchen mit Kohlen tüchtig im Wasser geschüttelt war. Sodann wurde das Wasser längere Zeit im Kochen erhalten, das Körbchen in demselben öfter geschüttelt, und das Gewicht nach dem Erkalten bestimmt. Der Gewichtsverlust, welchen das Messinggefäss leer unter gleichen Bedingungen erlitt, war vorher durch mehrere Versuche festgestellt worden.

Folgende Werthe wurden erhalten:

Kohle I.

a.

Spec. Gewicht der gashaltigen Kohle : 1,27

" " Koble nach dern Kochen : 1,26

b.

Spec. Gewicht der frisohen Kohle : 1,28

" " " Kohle nach dem Kochen : 1,29

" " " Kohle n. nochm. Kochen : 1,29

Kohle II.

a.

Spec. Gewicht dor frischen Kohle : 1,314

" entgasten Kohle : 1,309

b.

Spec. Gewicht der friseben Kohle : 1,297

, , , entgasten Kohle : 1,301 
414 v. Meyer: Untersuchung der aus einigen

Kohle III.

Spec. Gewicht der frischen Kohle : 1,332

" ,, entgasten Kohle : 1,335

Der Umstand, dass sich kaum ein Unterschied zwischen dem spec. Gewicht der gashaltigen und dem der von Gasen möglichst befreiten findet, verliert das Auffallende, wenn man annimmt, dass die Klüfte und Sprünge, durch welche das Gas entweicht, so fein sind, dass das Wasser nicht eindringen kann. Daraus würde zu folgern sein, dass eine Kohle nie vollständig in siedendem Wasser entgast werden kann.

Vor Abschluss dieser Mittheilung wird es zweckmässig sein, sümmtliche analytische Resultate ïbersichtlich zusammenzustellen:

\begin{tabular}{|c|c|c|c|c|c|c|}
\hline $\begin{array}{l}\text { Nammer der Anaiysen. } \\
\text { Zeit der Verarbeitung. }\end{array}$ & $\mathrm{CO}_{2}$ & $\mathrm{CH}_{4}$ & $\mathrm{C}_{2} \mathrm{H}_{6}$ & 0 & $\mathbf{N}$ & $\begin{array}{l}\text { 10oGrm. } \\
\text { lieferten } \\
\text { Gas Cc. }\end{array}$ \\
\hline $\begin{array}{l}\text { Grube Kronprinz } \\
\text { A. Wahlschieder. Flötz }\end{array}$ & & 23,06 & & & 60,85 & \\
\hline Bläser (1) & 0 & 23,57 & $\cdots$ & 16,09 & 60,34 & \\
\hline Grubenluft (2) und (3) & 1,25 & 2,39 & - & 17,77 & 78,59 & \\
\hline & & 2,65 & - & 15,17 & 78,13 & \\
\hline (4) (2 Wochen) & 8,51 & 63,86 & 3,88 & 0,25 & 23,50 & - \\
\hline (5) $(10$ Wochen $)\}$ & 33,66 & 24,08 & 4,72 & 0,81 & 36,73 & 42,0 \\
\hline (6) (2 Wochen) & 4,85 & 72,88 & - & 0 & 22,27 & - \\
\hline Schwalbacher Flötz. & & & & & & \\
\hline $\begin{array}{l}\text { (7) (3 Wochen) } \\
\text { (8) (5 bis } 6 \text { Wochen }\end{array}$ & $\begin{array}{r}4,57 \\
49,95\end{array}$ & $\begin{array}{r}51,84 \\
\quad(15\end{array}$ & $\begin{array}{l}5,92 \\
04)\end{array}$ & $\begin{array}{l}0,26 \\
1,39\end{array}$ & $\begin{array}{c}37,41 \\
(33,62)\end{array}$ & $\overline{32,0}$ \\
\hline (9) (3 bis 4 Wochen) & 38,05 & 15,06 & 13,06 & 1,16 & 32,40 & 19,0 \\
\hline (10) (5 Wochen) & 29,50 & 12,44 & 9,48 & 3,09 & 45,49 & 25,0 \\
\hline (11) (4 Wochen) & 15,22 & 52,12 & - & 0,56 & 32,10 & 22,5 \\
\hline Grube Geis lautern. & & & & & & \\
\hline Flätz Nr.4 (12) (6 Woch.) & 19,19 & 37,94 & 3,22 & 0,97 & 38,68 & 26,0 \\
\hline Flötz Nr.5 (13) (8 Woch.) & 35,66 & 9,46 & 3,61 & 2,63 & 48,64 & 21,2 \\
\hline Flötz Nr. 6 (14) (6 Woch.) & 26,12 & 55,65 & $\cdots$ & 1,20 & 17,03 & 53,0 \\
\hline Grube Gerhaxd. & & & & & & \\
\hline Schlagendes Wetter (15) & 0,62 & 22,52 & - & 14,01 & 62,85 & - \\
\hline Fl. Anna (16) & Spur & 91,76 & - & - & 8,24 & 188,0 \\
\hline "Sophie (17)! & 1,83 & 90,79 & - & - & 7,38 & 158,0 \\
\hline "Max (18) & 2,90 & 89,93 & - & - & 7,27 & 180,0 \\
\hline
\end{tabular}


lch glaube durch die oben mitgetheilten Versuche, die Bischof'schen Analysen als in hobem Grade unwahrscheinlich hingestellt za haben. Seine Methode, durch Absorption mit Chlor die sehweren Kohlenwasserstoffe $z u$ bestimmen, konnte keine genauen Resultate ergeben. Die Abwesenheit von Kohlenwasserstoffen der Reibe $\mathrm{C}_{n} \mathrm{H}_{2 \mathrm{n}}$ ist nach meinen Analysen constatirt. Das Auftreten solcher Gase bei Grubenbränden ist noch zu beweisen.

Eine andere Frage ist die, $o b$ in den von Bisch of untersuchten Grubengasen möglicherweise eine geringe Menge Aethylwasserstoff enthalten war. Obwohl ich in den verschiedenen Grubenexhalationen (s. Anal. (1) (2) (3) und (15)) niemals diesen Gemengtheil gefunden habe, ist doch, da derselbe in einigen Kohlen auftritt, die Möglich. keit seines Vorkommens in den Grubengasen nicht ausgeschlossen.

Die Beziehungen zwischen Gasmenge und Gehalt an Grubengas sind schon bei den Kohlen der Grube Gerhard (Anal. (16), (17), (18)) erörtert worden.

Kohlensäure und Kohlenwasserstoffe ergänzen sich gegenseitig wenigstens annähernd, sie sind in gewisser Weise complementiär. Dies Verhalten findet die einfachste Erklärung dadurch, dass Kohlensäure durch Oxydation der Kohlenwasserstoffe, besonders des Grubengases, entsteht. (Vergl. Anal. (4) und (5), (7), (8)).

Während die Kohlen der Gruben Kronprinz und Geislautern mehr oder weniger das Gepräge der Verwitterung an sich tragen, welche sich (ausser in den Verwitterungsproducten) in der schnellen Zunahme des Kohlensäuregehalts der Gase abspiegelt, zeigten die Kohlen der Grube Gerhard, obwohl sie 4 bis 5 Wochen gelegen hatten, keine Spur der Verwitterung. Ihre Härte und unregelmässige Structur unterscheidet sie erheblich von den Kohlen der beiden anderen Gruben, welche theilweise bröcklich, alle regelmässig geschichtet, dem Eindringen der Luft keinen Widerstand leisten konnten.

Die Oxydation der Kohlen in den Gruben trägt sicherlich dazu bei, die Luft in denselben zu verschlech- 
416 Schumann: Zur Bestimmung der Phosphorsäure.

tern. Abgesehen davon, dass ein Theil des Sauerstoffs ihr entzogen wird, erhält sie noch zum Ersatz für den Verlust ein irrespirables Gas zurück. - Durch die Analysen der Grubenluft [(2) und (3)] von einer vielleicht ausgezeichnet ungünstigen Oertlichkeit hoffe ich die Aufmerksamkeit der Bergbeamten auf die Nothwendigkeit einer gründlichen Untersuchung dieser Verbältnisse geleitet $z u$ haben.

Meine Bemühungen, zwischen Lagerungsverhältnissen und Gasgehalt der Kohlen Beziehungen aufzufinden, sind auch diesmal vergeblich geblieben; von grösserer Bedeutung für diese Verhältnisse ist jedenfalls, wie ich oben hervorhob, die Structur. Auch die Frage, ob das ,zu Tage Ausgehei" " der Flötze einen Einfluss anf die Qualität und Quantität der eingeschlossenen Gase ausübe, habe ich gepruft: jedoch konnte ich keine prägnanten Unterschiede auffinden. Nach der bergmännischen Erfahrung, dass häufig Flötze, welche zu Tage ausgehen, von Schlagwettern frei sind, hätte man Differenzen im Gasgehalt der, verschiedenen Flötzen entnommenen, Kohlen erwarten dürfen. Jedoch liegen andererseits Beobachtungen entgegengesetzter Art vor, so dass keinenfalls das Moment des zu Tage Ausgehens das einzige ist, welches die Befreiung von schlagenden Wettern bedingt.

Leipzig, Laboratorium von Prof. Kolbe.

\section{Zur Bestimmung der Phosphorsäure; von}

\section{Camillo Sohumann.}

Die folgenden Versuche wurden bereits im Jahre 1869 begonnen. Durch versehiedene Umstände an ihrer Fortsetzung behindert, wurde ich besonders durch 